\title{
ELM moderation with ICRF heating on JET
}

\author{
G P Maddison ${ }^{1}$, J A Snipes ${ }^{2}$, J-M Chareau ${ }^{1}$, G D Conway ${ }^{3}$, \\ I H Hutchinson ${ }^{2}$, L C Ingesson ${ }^{4,11}$, H R Koslowski ${ }^{5,11}$, A Loarte $^{6}$, \\ P J Lomas ${ }^{1}$, M J Mantsinen ${ }^{7}$, G F Matthews ${ }^{1}$, L Meneses ${ }^{8}$, M F F Nave ${ }^{8}$,

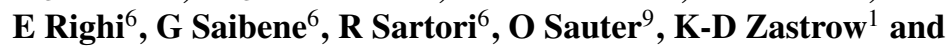 \\ contributors to the EFDA-JET Workprogramme ${ }^{10}$
}

${ }^{1}$ EURATOM/UKAEA Fusion Association, Culham Science Centre, Abingdon, Oxon OX14 3DB, UK

${ }^{2}$ MIT Plasma Science and Fusion Centre, Cambridge, MA 02139, USA

${ }^{3}$ Max-Planck IPP, EURATOM Association, D-85748 Garching, Germany

${ }^{4}$ FOM-IVP, EURATOM Association, Postbus 1207, NL-3430 BE Nieuwegein, Netherlands

${ }^{5}$ IPP, Forschungszentrum Jülich GmbH, EURATOM Association, D-52425 Jülich, Germany

${ }^{6}$ EFDA-CSU, D-85748 Garching, Germany

${ }^{7}$ Helsinki University of Technology, Association Euratom-Tekes, Finland

${ }^{8}$ CFN, EURATOM-IST Associação,1096 Lisbon, Portugal

${ }^{9}$ CRPP, Association EURATOM-Confédération Suisse, EPFL, 1015 Lausanne, Switzerland

E-mail: geoff.maddison@ukaea.org.uk

Received 16 January 2002, in final form 5 August 2002

Published 27 August 2002

Online at stacks.iop.org/PPCF/44/1937

\begin{abstract}
A high-density, ELM-free, 'enhanced D $\alpha$ ' (EDA) H-mode has been demonstrated with Ohmic or RF heating on the Alcator C-Mod device. An allied 'low particle confinement' $\mathrm{H}$-regime was also observed previously in 'Mk0' divertor experiments on JET. Initial attempts to recover similar states in the JET 'MkIIGB' divertor configuration are reported, employing appropriately shaped magnetic equilibria. Comparisons between cases with and without divertor cryopumping show enhanced edge sources tend to increase ELM frequency and reduce their size, without degrading confinement. However, torus pressure remains below that in C-Mod owing to lower wall recycling in JET. With cryopumping on, ion cyclotron resonance heated (ICRH)-dominated combined heating may be adapted for maximum magnetohydrodynamic stability, leading to recurrent ELM-free H-mode phases. Core confinement peaks as density and edge radiation rise in each ELM-free phase, while only small compound ELMs occur between them. Moreover magnetic and density fluctuations in each quiescent period are concentrated around two frequencies, although these modes appear to rotate in the opposite direction to coherent modes seen during EDA on C-Mod. Pedestal collisionality also remains much lower in both latest and even earlier LPCH JET cases. Finally an effect of heating

\footnotetext{
${ }^{10}$ See appendix of paper by J Pamela 2000 18th IAEA Fusion Energy Conf. (Sorrento, Italy, October 2000).

${ }^{11}$ Affiliations 4 and 5 are partners in the Trilateral Euregio Cluster (TEC). 
scheme itself on edge properties is indicated by alternative $\mathrm{H}$-modes at the same density and power, with lower pedestal electron temperature and higher ELM frequency occurring for ICRH than for NBH. Closer matches in dimensionless plasma variables would clarify scalability of EDA-mode and its potential as an integrated next-step regime.

\section{Introduction}

At present, the most developed approach to the high-performance conditions required in a burning next-step tokamak is based upon the divertor ELMy H-mode regime. On the other hand, its best performance tends to be accompanied by low-frequency, but large-amplitude, Type I ELM fluctuations which would extrapolate in a next-step to very severe intermittent loads on boundary surfaces [1]. Consequently to avoid erosion and risk of plasma contamination, ways to moderate ELMs without compromising confinement are a high priority for fusion plasmas. One technique under investigation involves deliberate addition of small amounts of a medium $Z$ impurity into the plasma edge, in order to disperse more efflux power as radiation [2-4]. An alternative scheme is implied by results on the Alcator C-Mod device, using strongly shaped plasmas with Ohmic or RF auxiliary heating [5-7]. At high density such discharges demonstrate a spontaneous transition to a state with energy confinement only $\approx 20 \%$ lower than in ELM-free H-mode, but quasi-stationary and having no or only modest ELMs. For lower $\beta_{\mathrm{N}}$ values, ELMs are absent altogether, and at higher $\beta_{\mathrm{N}}$, fluctuations with only mild energy expulsion emerge [7]. Instead edge recycling is substantially increased, prompting the description 'enhanced $\mathrm{D} \alpha$ ' (EDA) H-mode, while crucially a persistent, high-order quasicoherent mode (QCM) which is strongly localized in the edge appears to induce continuous rather than bursting relaxation of pedestal gradients $[6,7]$. This sustained efflux should then assist optimization of the energy to effective particle confinement time ratio $\left(\tau_{E} / \tau_{\mathrm{p}}^{*}\right)$ too, hence supporting steady control of impurities and ash exhaust in a next-step machine. Somewhat similar effects had also been observed in early operations on JET using its open 'Mk0' divertor geometry [8], which exploited target tiles on the top surface of the vacuum vessel. Naturally strong shaping in these plasmas generally promoted ELM-free H-modes. In RF-heated doublenull examples at high density, sudden interruption of gas fuelling or laser ablation of a radiating impurity led to a sudden decline of particle content and radiated power, while stored energy rose, thus defining a related 'low particle confinement' (LPC) H-mode [8].

The potential of EDA/LPC H-states for next-step use depends on their scaling from existing cases. Initial attempts to reproduce them in recent JET single-null divertor experiments are reported, focusing on pure or predominantly ion cyclotron resonance heated (ICRH) plasmas. Some matching results with neutral beam heating $(\mathrm{NBH})$ also allow the underlying question of H-mode sensitivity to heating scheme to be revisited [9]. As noted, shaping of the magnetic equilibrium is a key ingredient, and a new configuration was adopted in which reasonable elongation $(\kappa \approx 1.7)$ and high triangularity $\left(\delta_{1} \approx \delta_{\mathrm{u}} \geqslant 0.4\right)$ were adapted to the 'MkIIGB' arrangement in JET. This design incorporated an axisymmetric closed divertor within the torus, with a central barrier ('septum') between its inboard and outboard sides, and a cryopump outside its outboard lower corner [1,10]. A typical magnetic reconstruction by EFIT, within the first-wall contour, is illustrated in figure 1. Evidence from C-Mod indicates medium to high values of edge safety factor $q_{95} \geqslant 3.5$ are also important [7]. In other words, an existence region in terms of magnetic equilibrium parameters is suggested for EDA-modes at higher densities, as exemplified in figure 2, overlapping with ELM-free $\mathrm{H}$-modes at lower densities. Corresponding ranges spanned in average triangularity $\bar{\delta} \equiv\left(\delta_{1}+\delta_{\mathrm{u}}\right) / 2$ and $q_{95}$ over latest JET cases are 


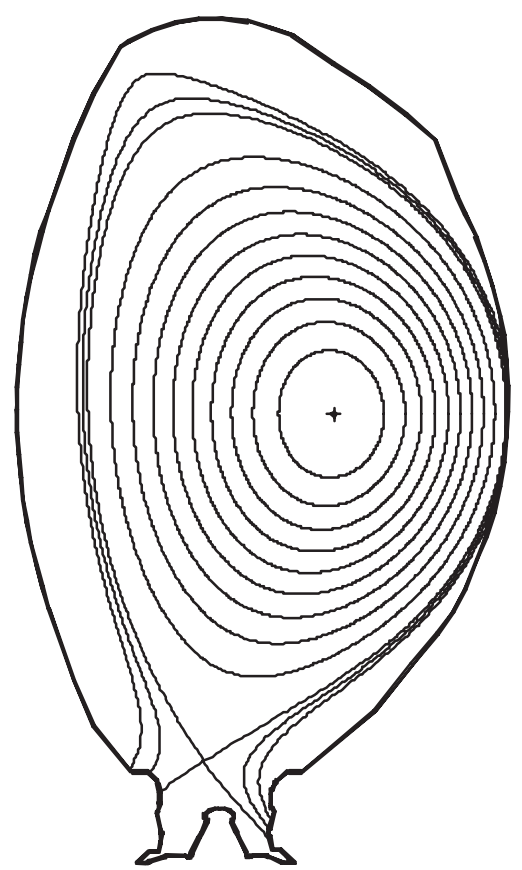

Figure 1. Strongly shaped plasma equilibrium adapted to the JET MkIIGB divertor geometry (EFIT reconstruction for pulse \#50677 at 24 s). The strike points are high relative to the cryopump duct entrance (bottom right-hand corner).

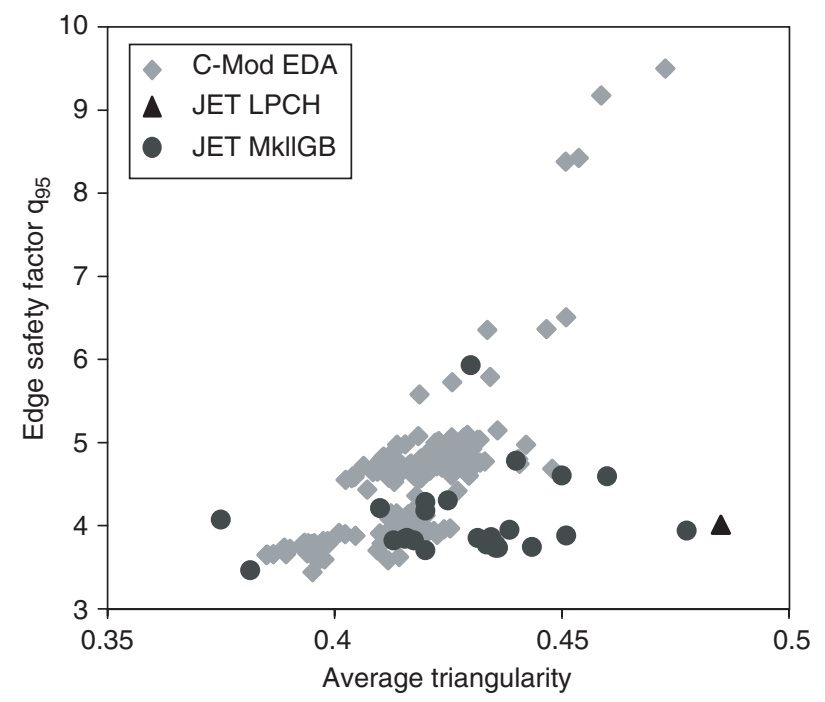

Figure 2. Magnetic equilibrium parameters for sample EDA-modes in Alcator C-Mod, for LPCHmode in JET Mk0 (\#23023), and values spanned over latest experiments in JET MkIIGB. Similar ranges are covered in each device.

superimposed in figure 2, confirming that values comparable to C-Mod EDA data were realized. Note that the MkIIGB plasmas generally had inboard and outboard strike points placed respectively high on the divertor vertical tiles and above the duct entrance to the cryopump, while the X-point was clear of the septum structure. These features lessened pumping from 
the divertor, strengthening peripheral neutral particle sources in the main torus, which are themselves a factor suspected to assist EDA transitions in C-Mod [5, 7]. In the next section, tests therefore maximizing torus gas pressure by eliminating cryopumping altogether are described. Most experiments were aimed at producing long ELM-free periods, however, since both EDA and LPC H-regimes develop out of such states [7,8]. A following section presents results in particular involving asymmetric phasing of ICRH to seek better magnetohydrodynamic (MHD) stability through more benign sawtooth behaviour. Key turbulence signatures of the emergence of EDA properties are pursued. Contrasting effects of alternative heating schemes are also briefly explored. Finally a summary and prospects for further investigation are discussed.

\section{Variation of edge particle sources}

The strongly shaped magnetic configurations prescribed for EDA-like conditions match very well to the ICRH antennae in JET, allowing excellent coupling of waves to the plasma even without feedback control on the resistance $R_{\mathrm{C}}$. First trials established good hydrogen-minority

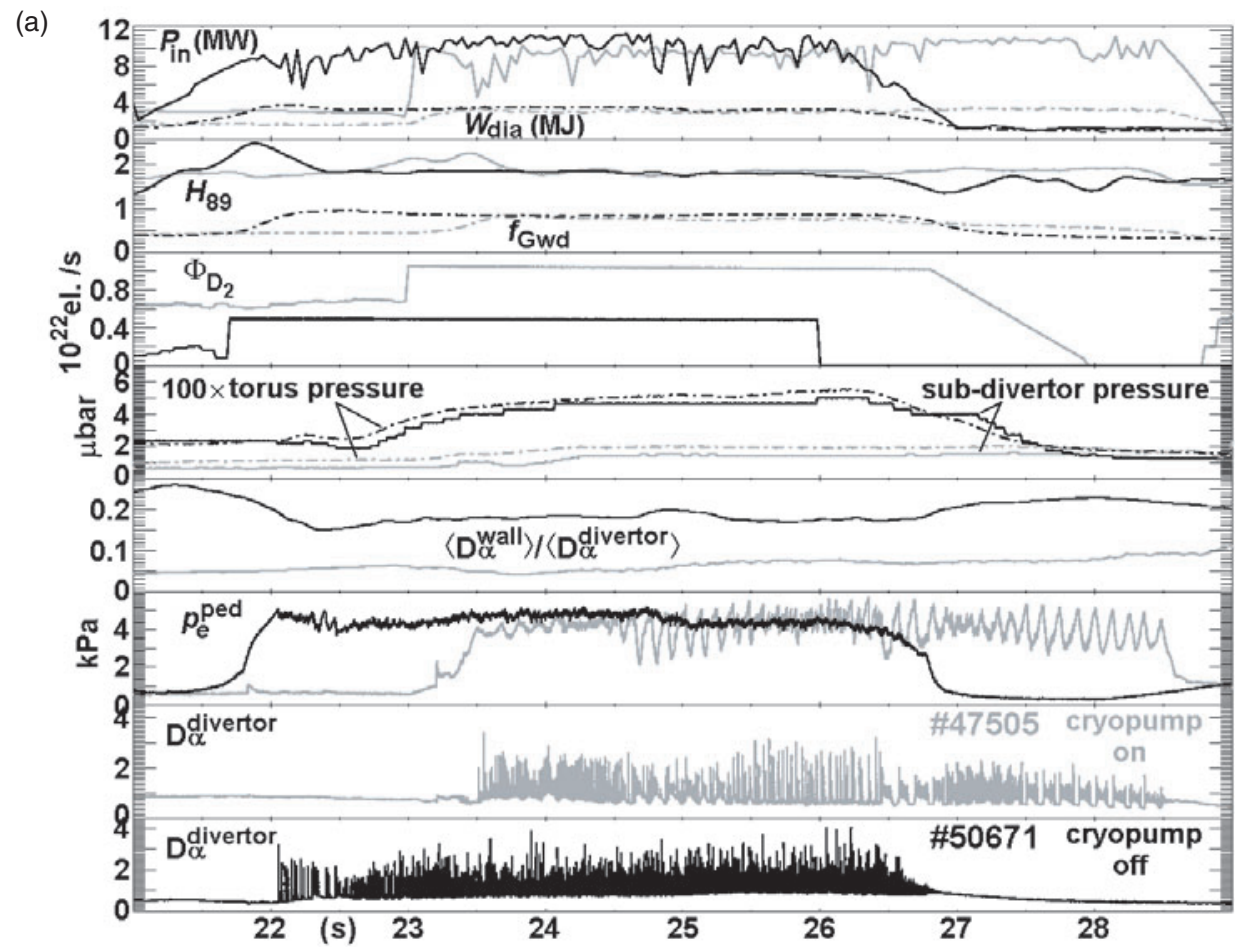

Figure 3. (a) Matching ICRH plasmas in JET MkIIGB with (\#47505) and without (\#50671) divertor cryopumping: total input power and diamagnetic stored energy; normalized confinement and density; gas inputs; Penning gauge measurements of gas pressure in the main torus $(\times 100)$ and sub-divertor volume; moving time-average ratios of total $\mathrm{D} \alpha$ photon production rates in the main torus to the divertor; estimated electron pedestal pressure from edge ECE plus interferometer channel at $3.74 \mathrm{~m}$; respective divertor $\mathrm{D} \alpha$ emission. The unpumped case has higher gas pressure and stronger torus particle sources, plus higher frequency ELMs for similar electron pedestal height. (b) Expanded intervals of ELMs and electron pedestal temperature (ECE) for the same pair of ICRH plasmas with (\#47505) and without (\#50671) divertor cryopumping. Compound to more distinct ELMs occur when the pump is on, while the unpumped case has more regular, higher frequency ELMs with smaller temperature perturbations. 

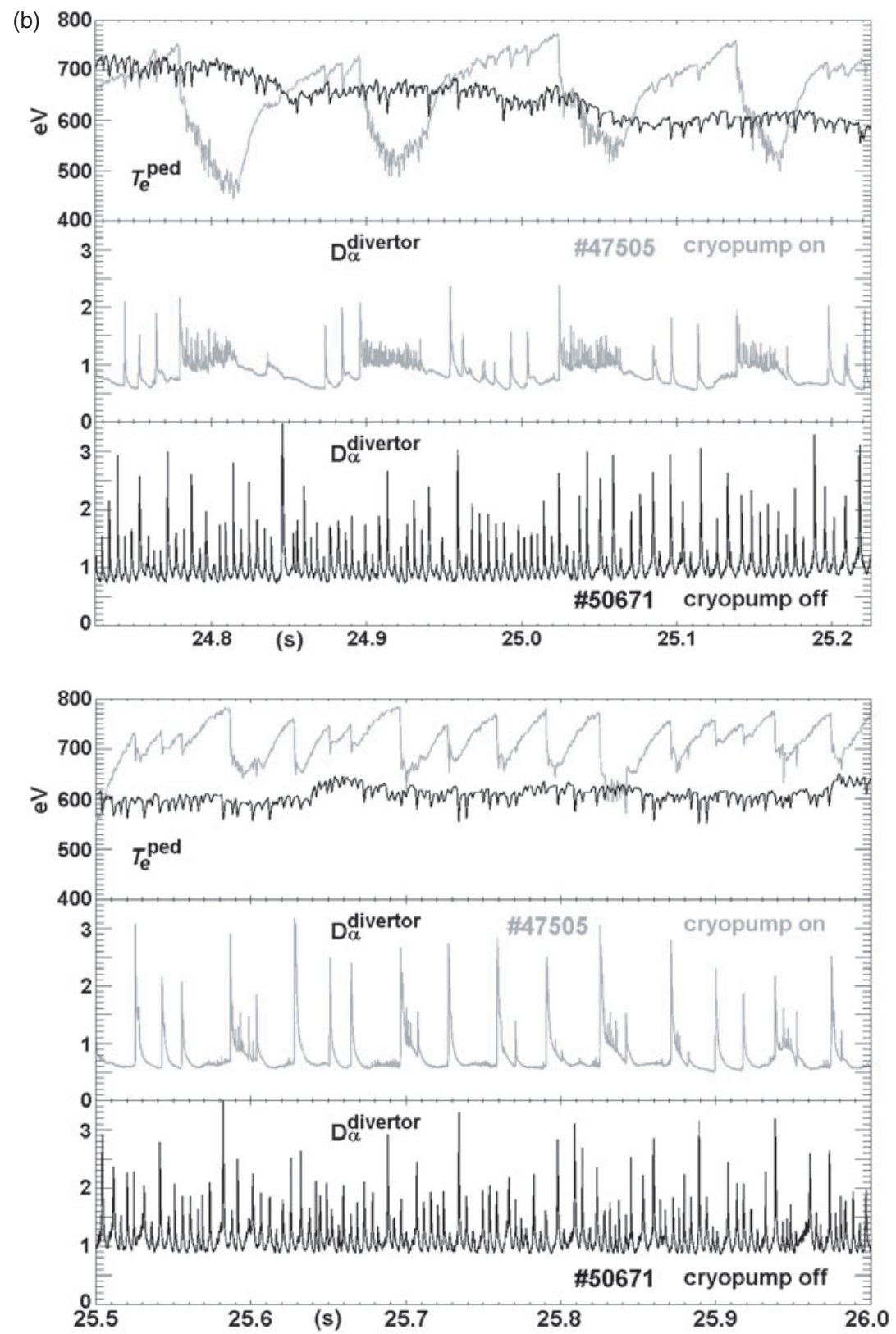

Figure 3. (Continued)

fundamental heating using a frequency of $42 \mathrm{MHz}$ at $B_{0}=2.7 \mathrm{~T}$ for on-axis resonance. However, they retained ELMs of moderate size and frequency often seen in RF-heated H-modes, i.e. no transitions to ELM-free behaviour were effected. All these plasmas had the divertor cryopump operating, whereas access to EDA states may actually be aided by elevated neutral gas pressure in the main torus $[5,7]$. Specifically to accentuate this property, succeeding experiments repeated similar discharges but with the cryopump switched off. Two 
matching pulses $\left(2.7 \mathrm{~T}, 1.7 \mathrm{MA}, q_{95} \approx 4.7, \delta_{\mathrm{u}} \approx 0.48\right)$ are contrasted in figure 3 , where $P_{\text {ICRH }} \approx 11 \mathrm{MW}$ was steadily coupled in each case. Note the pumped example $(\# 47505)$ had marginally smaller elongation and lower triangularity $\left(\kappa^{\mathrm{ON}} \approx 1.64, \delta_{1}^{\mathrm{ON}} \approx 0.35\right)$ compared with the unpumped one $\left(\# 50671, \kappa^{\mathrm{OFF}} \approx 1.69, \delta_{1}^{\mathrm{OFF}} \approx 0.42\right)$. Also to improve stability against MHD activity, ICRH was increased in two steps in the former, while the latter incorporated a brief period of NB pre-heating. Otherwise, their stored energies and performance in terms of confinement normalized to the ITERH89P L-mode scaling law [11] $H_{89} \equiv \tau_{E} / \tau_{H 89 \mathrm{P}}\left(H_{89}^{\mathrm{ON}, \mathrm{OFF}} \approx 1.8\right)$ were very similar. Normalizing density to the Greenwald limit $\left.f_{\mathrm{Gwd}} \equiv \pi\left(\bar{n}_{\mathrm{e}} 10^{20} \mathrm{~m}^{-3}\right)\left(a^{2} \mathrm{~m}^{2}\right) / I_{\mathrm{p}} \mathrm{MA}\right)$, a fraction $f_{\mathrm{Gwd}}^{\mathrm{ON}} \approx 0.6$ was obtained with the pump on, whereas $f_{\mathrm{Gwd}}^{\mathrm{OFF}} \approx 0.8$ was reached without cryopumping, even for less puffing. Neutral gas pressure in this case (\#50671) was indeed raised by a factor of $\approx 3$ in both the volume below the divertor plates and the main torus. Accompanying respective signals for total $\mathrm{D} \alpha$ photon production rates, time-averaged in a moving $\tau_{E} \sim 0.5 \mathrm{~s}$ window, show that majority ionization sources were almost doubled in the main chamber and actually lowered in the divertor. An approximately 3 times relative enhancement of torus particle sources compared with pumped quantities was consequently implied (figure 3(a)).

Again ELMs were not eliminated, but divertor $D \alpha$ emission reveals their character was strongly modified. Two magnified time intervals are traced in figure $3(b)$, showing that in the pumped discharge (\#47505) complex compound ELM signatures with a rising base level of emission sometimes occurred; then tended to more distinctly separated ELMs, with a frequency around $30-50 \mathrm{~Hz}$, at other times. Note that the former 'hashy' periods led to falls in stored energy, line-average density, and all electron pedestal quantities (detailed below). They are therefore unlikely to be related to Type II ELMs seen close to double-null configuration in ASDEX-U [12], or so-called 'mixed Type I + II' ELMs seen at lower $q_{95}$ in both ASDEX-U [12] and at high density for similar high $\delta$ in JET [13]. Recall that all of these cases involved $\mathrm{NBH}$, rather than ICRH as here. Throughout the unpumped plasma (\#50671) in figure 3, on the other hand, its ELMs remained regular and with comparable recycling amplitudes, but at much higher frequency, around $200 \mathrm{~Hz}$. Plasma pressure gradients in the pedestal region were not resolved in these experiments, but electron properties at the top of the pedestal may be estimated from the point of gradient change in ECE edge temperature profiles, together with an interferometer outer channel viewing along a chord at $3.74 \mathrm{~m}$ (relative to $R_{0}=2.96 \mathrm{~m}$, maximum $R_{\text {separatrix }}=3.86 \mathrm{~m}$ ) [14]. Pedestal density $n_{\mathrm{e}}^{\text {ped }}$ was slightly raised and temperature $T_{\mathrm{e}}^{\text {ped }}$ slightly lowered without pumping (\#50671), but such that the pressure height $p_{\mathrm{e}}^{\text {ped }}=n_{\mathrm{e}}^{\text {ped }} T_{\mathrm{e}}^{\text {ped }}$ was, on average, almost unchanged from the pumped reference (\#47505). Recall that the large $p_{\mathrm{e}}^{\text {ped }}$ excursions seen for the latter near 25 and $26.7 \mathrm{~s}$ in figure $3(a)$ are the result of multiple ELMs and not single fluctuations (cf figure 3(b)). Fractional drops in temperature $\left(\Delta T_{\mathrm{e}}^{\text {ped }} / T_{\mathrm{e}}^{\text {ped }}\right)$ for the more distinct ELMs around $25.7 \mathrm{~s}$ in each discharge were between 12-15\% (\#47505) and mostly 4-7\% (\#50671) respectively with and without pumping (figure $3(b)$ ), while inspection of ECE edge profiles also suggests their penetration depth into the unpumped plasma was correspondingly reduced. These comparisons therefore indicate main conductive losses [15] expelled in each ELM became smaller without pumping (\#50671) so that their sizes in terms of fractions of total plasma energy ejected $(\Delta W / W)$ (not directly resolved) probably decreased significantly. Although definite EDA behaviour did not emerge, this JET H-mode with elevated edge gas pressure thus accessed small, high-frequency ELMs but at high density and without loss of its good confinement.

Highest torus gas pressure obtained with the cryopump off, $\approx 0.05 \mu$ bar (\#50671), was $\approx 100$ times smaller than the sub-divertor pressure and still considerably lower than typical values for C-Mod plasmas, about $0.1-1 \mu$ bar. The latter reflects usually $100 \%$ recycling walls 
in C-Mod, so that its main chamber recycling can even dominate over divertor sources [16]. In contrast, vessel surfaces tend always to pump in JET, and as seen in figure 3( $a$ ) divertor recycling dominates even with the cryopump off. Their respective recycling regimes hence remained very different, possibly staying too low around the plasma for comparable impact in JET. A similar contrast had been noticed between earlier NB heated cases in the less closed JET 'MkIIA' divertor $[1,10]$ and ASDEX Upgrade, which again tends to exhibit stronger torus recycling $[17,16]$. For the former, separatrix plasma density rather than peripheral gas density chiefly governed confinement [10], whereas for the latter an inverse correlation between confinement and edge neutral particle abundance generally prevails [18]. Detailed modelling calculations are needed to analyse these differences, and to quantify possible effects of machine size/plasma scale lengths in relation to neutral particle mean free paths. Subsequent experiments reported here alternatively pursued EDA effects reverting to usual JET cryopumped operation.

\section{Long ELM-free periods}

Both LPCH in JET Mk0 and EDA in Alcator C-Mod are regimes emerging from ELM-free RF-heated H-modes [7,8]. A natural step was therefore to examine this state in MkIIGB (cryopump on) by tailoring the level of input power at slightly lower $q_{95}$ (lower points in figure 2). An initial obstacle for such plasmas is their liability to degradation by low-order MHD instabilities, particularly neoclassical tearing modes (NTMs). These are often encountered in JET H-modes owing to proximity of their onset critical $\beta_{\mathrm{N}}$ to the transition threshold [19]. The susceptibility is even aggravated in RF-heated cases, apparently as fast particle stabilization produces longer period sawteeth, which are then individually more capable of provoking adverse seed islands [19]. However, comparisons of conventional symmetric $(0+\pi 0+\pi)$ phasing of the ICRH antennae (as above) with alternative asymmetric $(-\pi / 2)$ phasing, plus slightly off-axis resonance, have suggested that the latter can lessen fast particle stabilization and so preserve more regular sawteeth [19]. This is exemplified for strongly shaped plasmas in figure 4 , where symmetric (\#53259, $\left.2.7 \mathrm{~T}, 2 \mathrm{MA}, q_{95} \approx 3.7, \bar{\delta} \approx 0.44\right)$ and asymmetric (\#53421,\#53422, 2.8 T, 2 MA, $q_{95} \approx 3.9, \bar{\delta} \approx 0.44$ ) phasing of ICRH at $42 \mathrm{MHz}$ are compared. Note that combined heating was also sustained throughout to maintain plasma rotation and inhibit any mode locking, but still with predominant $P_{\mathrm{ICRH}} \approx 6 \mathrm{MW}$. In the symmetric case (\#53259), a slightly lower input power resulted from a partial recovery of ICRH after an early drop, together with a step down to $P_{\mathrm{NBH}}=1 \mathrm{MW}$. Nevertheless, its $\beta_{\mathrm{N}}$ recovered to $\approx 1.7$ too until a delayed sawtooth prompted growth of $n=1,2$ magnetic modes, leading in turn to a fall in confinement, density and $\beta_{\mathrm{N}}$. Note that large ELMs were precipitated at each loss of normalized pressure. In one asymmetric example (\#53422, $\left.P_{\mathrm{NBH}} \approx 4 \mathrm{MW}\right)$, a delayed sawtooth similarly occurred soon after entering $\mathrm{H}$-mode, and led to rapid increases in $n=1,2$ activity, here signalling an NTM, which caused the pulse to terminate. The second asymmetric phasing discharge (\#53421, $P_{\mathrm{NBH}} \approx 4 \mathrm{MW}$ before $22 \mathrm{~s}, 2.5 \mathrm{MW}$ after $23 \mathrm{~s}$ ), on the other hand, demonstrated the capability to keep higher frequency sawteeth throughout and thence to remain free of magnetic disturbances. This feature of JET plasmas in fact contrasts with EDA-modes in C-Mod, where sawteeth are less prone to stabilization at its high absolute densities but typically have at least very large amplitudes [6]. Their conditions might therefore be staying lower with respect to the associated critical value of $\beta_{\mathrm{N}}$ [20].

When MHD modes were avoided by asymmetric phasing of ICRH, higher plasma performance could be maintained, as at larger $q_{95}$ exemplified in the preceding section. The quiescent plasma obtained (\#53421) then produced repetitive ELM-free H-mode intervals, as seen in figure 5, each one separated by strikingly small ELMs (see also figure 9). During each ELM-free interval, total stored energy $W$ rose to a peak, then began to decline again 


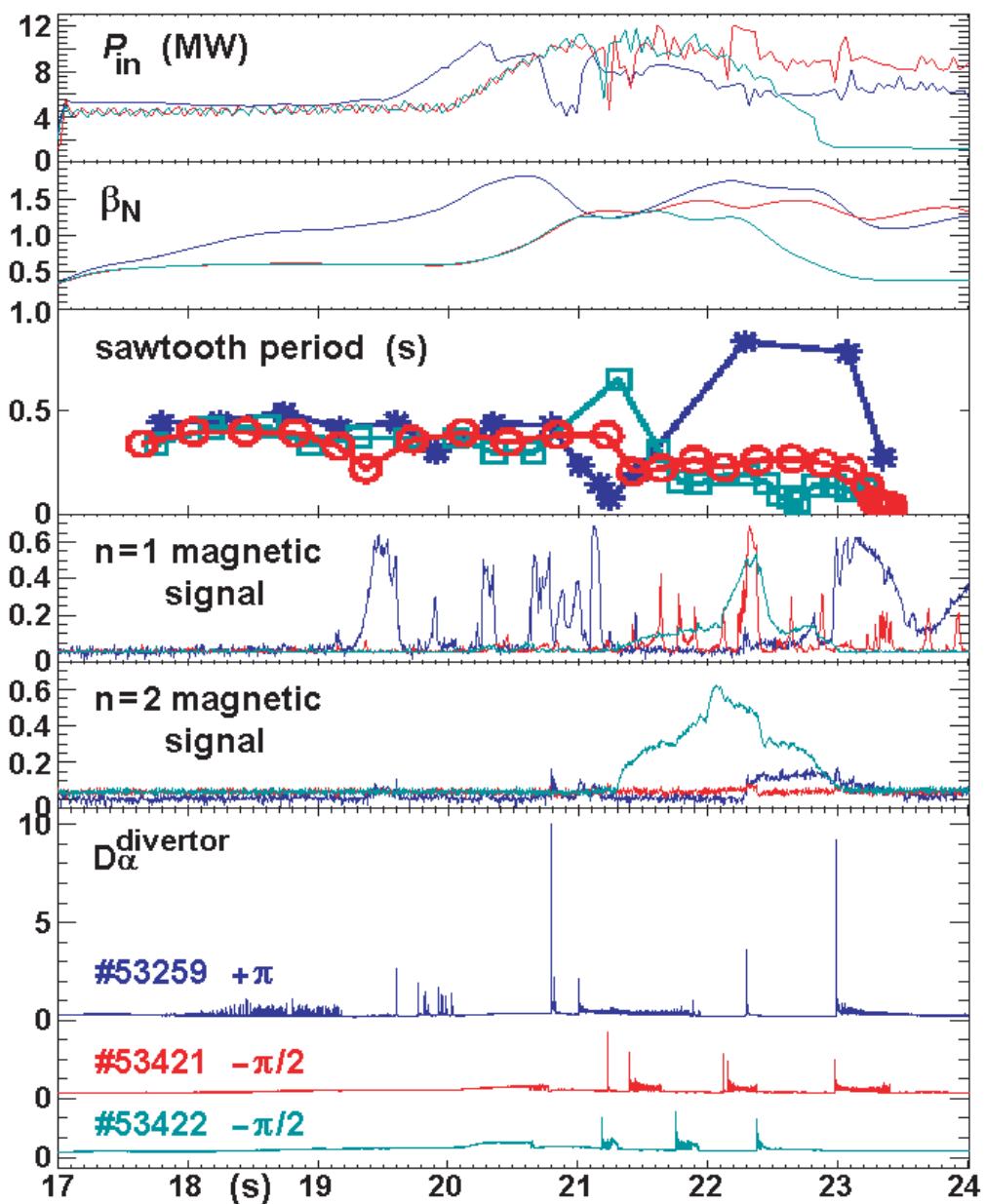

Figure 4. Input power, normalized pressure, sawtooth period, odd/even low-order magnetic signals and $\mathrm{D} \alpha$ emission for combined heating cases with symmetric $(0+\pi 0+\pi)$ phasing $(\# 53259)$ or asymmetric $(-\pi / 2)$ phasing (\#53241, \#53422) of ICRH. Slower sawteeth tend to stimulate MHD modes, while faster sawteeth throughout (\#53421) encourage freedom from magnetic activity.

while density and radiation fraction continued to increase steadily towards $f_{\mathrm{Gwd}} \approx 0.7-0.8$, $f_{\text {rad }} \approx 1$. Charge-exchange recombination spectroscopy (CXRS) data were not available for this pulse, but calculations with the PION code have been undertaken for related symmetric phasing cases where such ion temperature measurements were made. When ELM-free density rises occurred in these discharges, fast particle fractions rapidly collapsed, falling below $20 \%$ already at peak confinement. These cases hence support a similar finding with PION for pulse \#53421 approximating $T_{\mathrm{i}}(r, t)=T_{\mathrm{e}}(r, t)\left(T_{\mathrm{i}}(t) / T_{\mathrm{e}}(t)\right)^{\mathrm{XCS}}$, where electron temperature profiles were measured by LIDAR Thomson scattering and the ratio 'XCS' was determined at a single radius $r$ from an x-ray crystal spectrometer. Moreover tomographic reconstructions from multiple bolometer lines-of-sight indicate each radiation maximum built up mainly in the plasma periphery rather than the core, i.e. the latter was not being obviously diluted. Part of the decline in $W$ later in each ELM-free phase in figure 5 may consequently reflect that in fast particle population, but in combination with a genuine saturation or even a roll over of thermal energy confinement for steadily rising density as well. Eventually a burst 


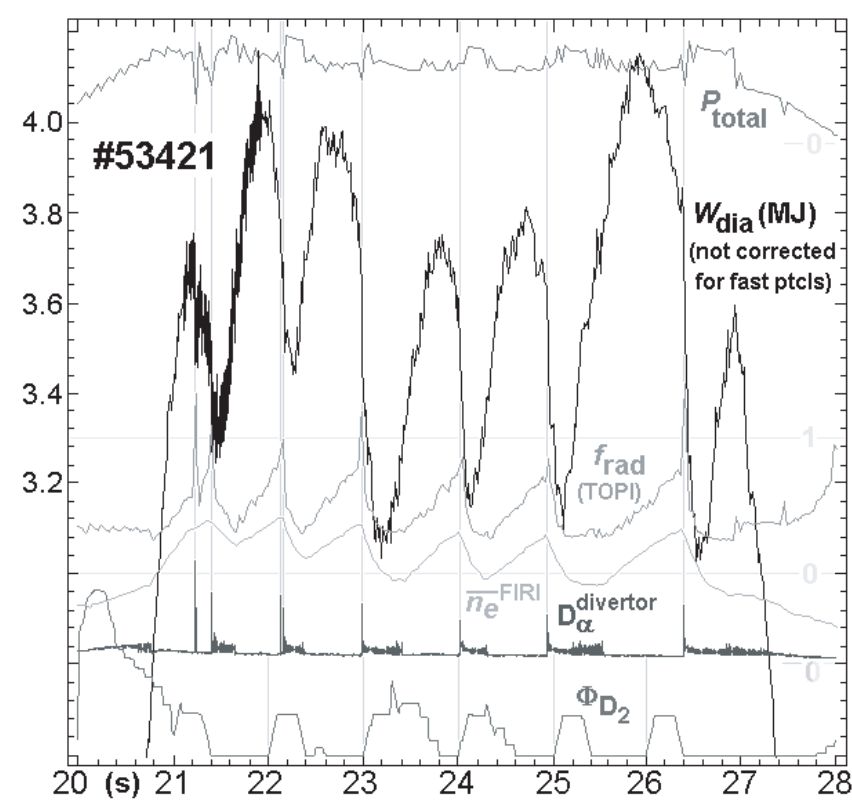

Figure 5. JET plasma (\#53421) with combined ICRH (asymmetric phasing) + lower NBH, exhibiting repeated ELM-free intervals. Main trace and scale: diamagnetic stored energy (including fast particles); other traces proportional to total heating power, radiated power fraction, line-average density, divertor $\mathrm{D} \alpha$ emission and $\mathrm{D}_{2}$ gas input. Stored energy rolls over before the end of each ELM-free cycle, while radiation and density continue to rise until the subsequent small ELM. Gas puffs had no effect at the levels applied.

of small, compound ELMs was triggered (see section 4 later), at which the electron pedestal immediately fell (figure 9), radiation rapidly decreased, and density dropped back slightly more slowly. Confinement meanwhile began to recover during the small ELMs, until they decreased in frequency and stopped, and the cycle repeated again. Even the largest first ELM in each burst is found from fast diamagnetic loop measurements to have only ejected total energy fraction $(\Delta W / W) \approx 3 \%$, perhaps consistent with the strong edge radiation simultaneously emitted. Recall this plasma did not involve any impurity 'seeding' [3,4] to reach these conditions, however. While it remained unsteady, it may therefore be approaching a desired regime of high density with small, tolerable ELMs. Short puffs of gas into the recurrent phases (figure 5), prompted by the technique accessing LPC H-mode [8] in JET Mk0, did not influence them at the levels tried. Also preliminary attempts to raise the plasma density prior to entering $\mathrm{H}$-mode, as induces EDA transition [7] in C-Mod, did not yet achieve a significant increase. This remains an aim for further experiments.

A defining feature of EDA-mode probably underlying its stationarity in C-Mod is a persistent quasi-coherent mode (QCM) fluctuation, which is concentrated both in a narrow edge region and in frequency, around $\sim 100 \mathrm{kHz}$, with intermediate poloidal and toroidal periodicities $m>30, n \approx 15-20[6,7]$. Its exact nature and hence scaling remain under study $[21,22]$, but it is observed to transform into fluctuations over a broad band of lower frequencies, in conjunction with small, 'grassy' ELMs, as pedestal temperature and pressure gradient increase [22]. Recently, activity quite reminiscent of this seems to have been detected between ELMs of so-called 'mixed' type in JET NBH plasmas at high density and triangularity [13]. A sample analysed spectrum for fast magnetic pick-up coils in the foregoing JET pulse (\#53421) is given in figure 6. Noticeably this reveals two dominant, narrow-band components between 
about 30 and $60 \mathrm{kHz}$, which were constant through its ELM-free periods but suppressed during ELMs. Furthermore, measurements with a four-channel X-mode reflectometer show that a pair of coherent density fluctuations again rose in intensity between bursts of ELMs, and became correlated in a similar frequency range (figure 6). These oscillations are clearest on its edge channels at $75,92,96 \mathrm{GHz}$, implying their location within the outer $<20 \%$ of the minor radius. A more detailed representation is depicted in figure 7 using a companion ten-channel O-mode reflectometer, but now plotting cross-power spectrum between two channels at $29.1 \mathrm{GHz}(\# 3$ ) and $50.5 \mathrm{GHz}$ (\#7) directly to incorporate a dependence on spatial extent. Density profiles from LIDAR Thomson scattering again indicate that these waves were reflected in the outer $\sim 10 \%$ of the plasma cross-section. Recall that loss of phase information in high frequency ELMs would reduce the corresponding plot effectively to white noise with reflected power spread evenly over a broad band of frequencies $(\leqslant 80 \mathrm{kHz})$. During the ELM-free period in figure 7 , however, a continuous concentration in two coherent peaks $\approx 25$ and $50 \mathrm{kHz}$ is again evident. Note since $f_{\text {rad }}$ rose close to unity, these discrete fluctuations were apparently not yet releasing much plasma energy. A toroidal mode number analysis of preceding magnetic signals also indicates values $n \approx+5$ to +10 , i.e. their sign denoting rotation in the ion diamagnetic drift direction. This is in contrast to QCMs in C-Mod, which instead rotate in the electron direction. In addition, the pair of JET modes here seems distinct from so-called 'washboard' modes commonly seen before between ELMs in JET H-regime [23], since these latter not only rotate again in the electron direction, but typically involve many more coherent frequency stripes in their spectra. More data are required to determine whether the signatures in pulse \#53421 are specific to its conditions, or might occur more generally in RFH or other ELM-free H-modes. It has also to be tested whether they can be strengthened, and then lead to a steady state.

Scaling of EDA behaviour between devices fundamentally must depend on respective dimensionless plasma variables. The foregoing turbulence effects also suggest that values in the pedestal region are likely to be essential. In fact, properties in representative high-field

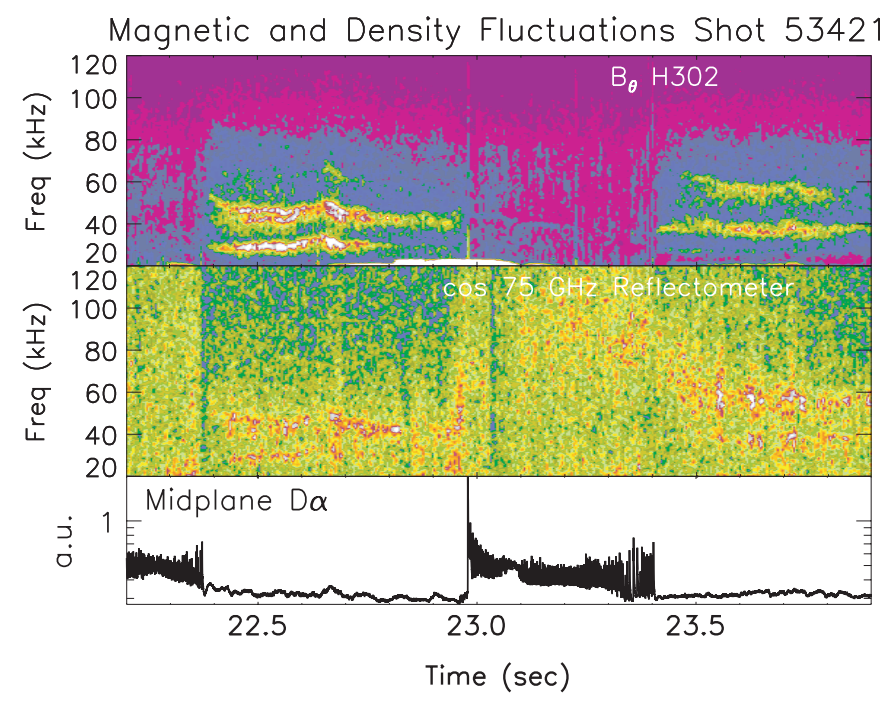

Figure 6. Spectra of fast magnetic pick-up coil (top), and $75 \mathrm{GHz}$ channel of an X-mode reflectometer (middle), together with mid-plane $\mathrm{D} \alpha$ signal (bottom), during combined heating plasma (\#53421). Coherent magnetic fluctuations at two frequencies between about $30-60 \mathrm{kHz}$ emerge during ELM-free but not ELMy periods. A similar pair of correlated density fluctuations also builds up in the ELM-free phases, suggesting regular activity in the plasma edge region. 


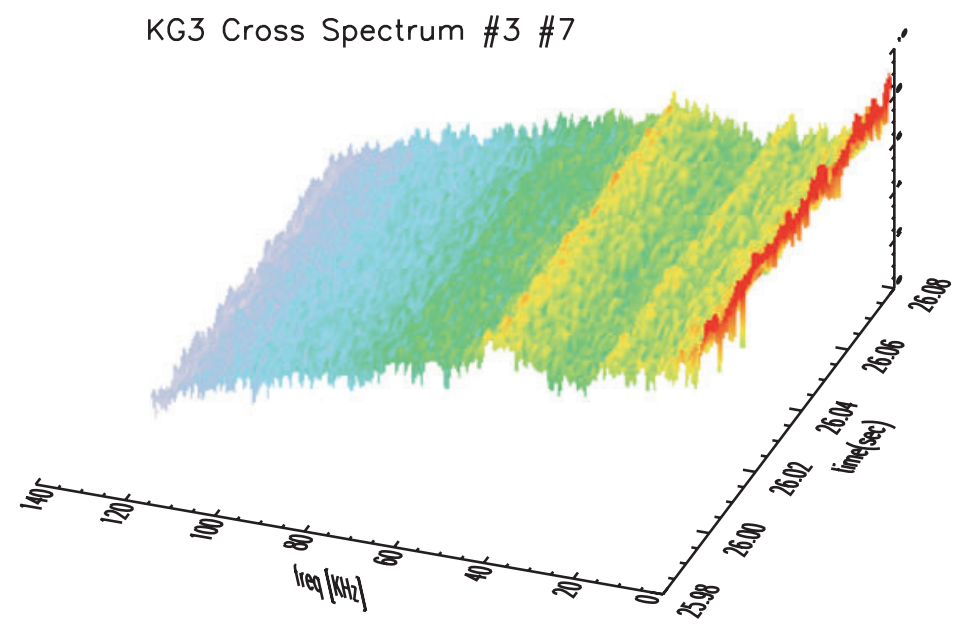

Figure 7. Fast cross-power spectrum between two channels at 29.1 and $50.5 \mathrm{GHz}$ of an O-mode reflectometer during an ELM-free phase of combined heating pulse (\#53421). Note that the vertical axis is logarithmic and spans ten orders of magnitude. Two concentrated edge modes around $\approx 25-50 \mathrm{kHz}$ are again evident.

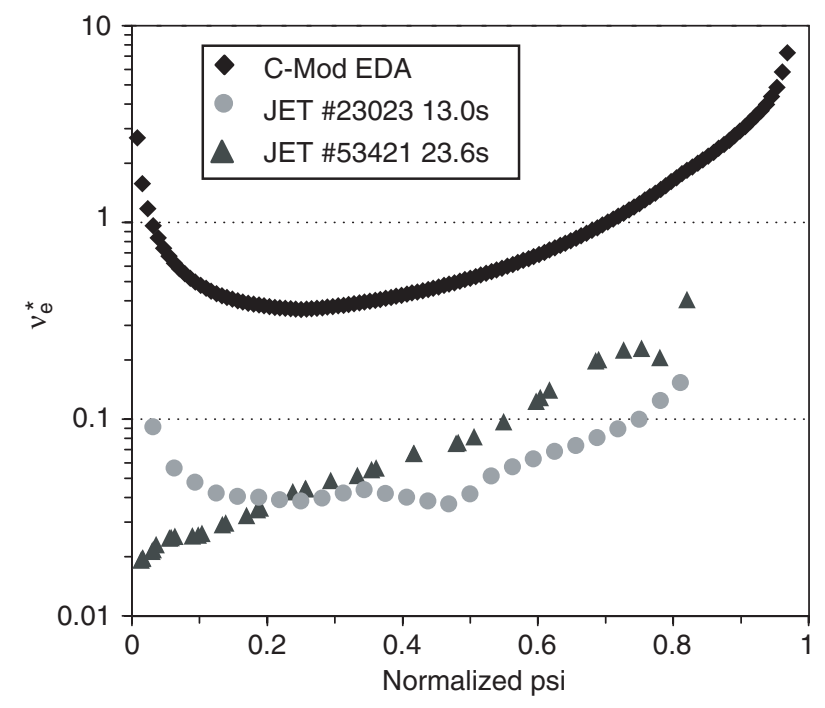

Figure 8. Profiles of electron collisionality $v_{\mathrm{e}}^{*}$ in a sample Alcator C-Mod EDA-mode, just prior to globally similar LPCH-mode in JET Mk0 (\#23023) and during ELM-free H-mode in JET MkIIGB with combined heating ( $\$ 53421$ ). Pure plasmas are assumed. C-Mod values are marginally collisional in the pedestal vicinity, whereas both JET pulses imply collisionless conditions.

Alcator C-Mod and larger, hotter, medium-field JET experiments resulted in edge normalized Larmor radii $\rho_{a}^{*}$ which were within a factor of $\approx 2$ when the collisionalities were closest $\left(1.5 \times 10^{-3} \approx \rho_{a}^{* \mathrm{JET}}<\rho_{a}^{* \mathrm{C}-\mathrm{Mod}} \approx 3 \times 10^{-3}\right)$ [24]. On the other hand, the latter electron collisionalities $\nu_{\mathrm{e}}^{*}$ tended to remain farther apart, as exemplified in figure 8, based on EFIT equilibria and assuming pure plasmas. Note $\nu_{\mathrm{e}}^{*}$ is likely to be important not least owing to its effect on edge bootstrap current, and so on MHD stability in the pedestal region. In JET, LIDAR density and temperature measurements are taken across the whole plasma cross- 
section, allowing $v_{\mathrm{e}}^{*}$ profiles to be derived separately from their inboard and outboard sides. These obviously ought to coincide as functions of normalized radius. Points shown in figure 8 are therefore the mean of these two curves, excluding data where they departed by $>40 \%$, viz for larger normalized radii. The C-Mod EDA and JET ELM-free (\#53421) states clearly lay in marginally collisional and collisionless regimes, and in particular their pedestal sections probably retained this disparity. Matching estimates with an earlier equilibrium code IDENTC of $v_{\mathrm{e}}^{*}$ just before entry into LPCH-mode in JET Mk0 (\#23023) reveal similar collisionless conditions, within uncertainties. Despite its resemblance to some global features of EDA, therefore, LPCH actually may not be fundamentally the same type of regime. Equally, ELMfree plasma properties here (\#53421) may not yet be reaching the domain for EDA behaviour. Further evidence for the importance of collisionality has been obtained on the DIII-D tokamak, where similar edge-localized coherent fluctuations to the QCM in C-Mod were observed for non-dimensionally identical pedestal conditions [25]. For collisionless edge plasmas, however, an alternative ELM-free $\mathrm{H}$-mode emerged in which very low frequency $(\approx 5 \mathrm{kHz})$ oscillations were detected around the foot of the pedestal [26]. These modes were of very low toroidal order $n=0-2$ too, consequently looking distinct from both QCMs in C-Mod and the JET ELM-free signatures described above (\#53421). Future experiments in JET will pursue non-dimensional identity to EDA plasmas directly to address the effect of collisionality.

\section{Effects of heating scheme}

Initial NB-heated pulses in the same highly shaped MkIIGB configuration, together with pure $\mathrm{RF}(0+\pi 0+\pi \mathrm{ICRH})$ and combined $(-\pi / 2 \mathrm{ICRH})$ heating cases above, provide a set of plasmas comparing additional power schemes for otherwise nearly fixed parameters. Three instances with approximately identical total input $\left(P_{\text {in }} \sim 10 \mathrm{MW}\right)$ from their respective methods are superimposed in figure 9. Furthermore, gas puffing was excluded from the NBH plasma, and only moderate levels $\left(\leqslant 10^{22}\right.$ electrons s $\left.{ }^{-1}\right)$ applied to the other two. Each one achieved similar density $f_{\mathrm{Gwd}} \approx 0.75$ and also performance, as measured by normalized confinement $t H_{89} \sim 2$. Very noticeably, though, their accompanying ELMs in terms of divertor recycling light were radically different: archetypal Type I bursts at a frequency of $10-15 \mathrm{~Hz}$ with NBH (\#47509, $2.7 \mathrm{~T}, 1.7 \mathrm{MA}, q_{95} \approx 4.8$ ); smaller, higher frequency ELMs for pure symmetric phasing ICRH (\#47505, 2.7 T, 1.7 MA, $q_{95} \approx 4.7$, cf section 2 ); virtually ELM-free behaviour, as discussed previously, under combined heating incorporating asymmetric phasing ICRH (\#53421, 2.8 T, 2.0 MA, $q_{95} \approx 3.9$, cf section 3 ).

A full account of edge stability against underlying localized modes would require determination of pedestal height, width and pressure gradient for both electrons and ions, edge current density and plasma rotation, all of which are capable of affecting the plasma response [27]. In the absence of complete data, though, it is again interesting to examine electron properties at the top of the pedestal using ECE plus interferometer signals as above. Traces in figure 9 show that $n_{\mathrm{e}}^{\text {ped }}$ was slightly higher with pure ICRH (\#47505) than with pure NBH (\#47509), but accompanying peak $T_{\mathrm{e}}^{\text {ped }}$ just before an ELM was considerably lower ( $\approx 780 \mathrm{eV}$ and $\approx 1 \mathrm{keV}$, respectively, around $26 \mathrm{~s}$ ). Recall a minimum pedestal temperature for Type I ELMs has been implied before [14,28], but here confinement is decreased by only $\approx 10 \%$ with ICRH compared to the hotter NBH edge. Furthermore, electron pedestal heights in ICRH H-modes have generally been found to reach about the same Type I pressure boundary at the start of ELMs as in pure or mainly NBH states [29]. Here resulting electron pedestal pressure peaks were roughly 10-15\% lower for RF than for NB heating, and ELM perturbations fell to $\left(\Delta T_{\mathrm{e}}^{\mathrm{ped}} / T_{\mathrm{e}}^{\mathrm{ped}}\right) \approx 12-15 \%(\# 47505, \sim 26 \mathrm{~s})$ from the characteristic $60-65 \%$ for Type I 


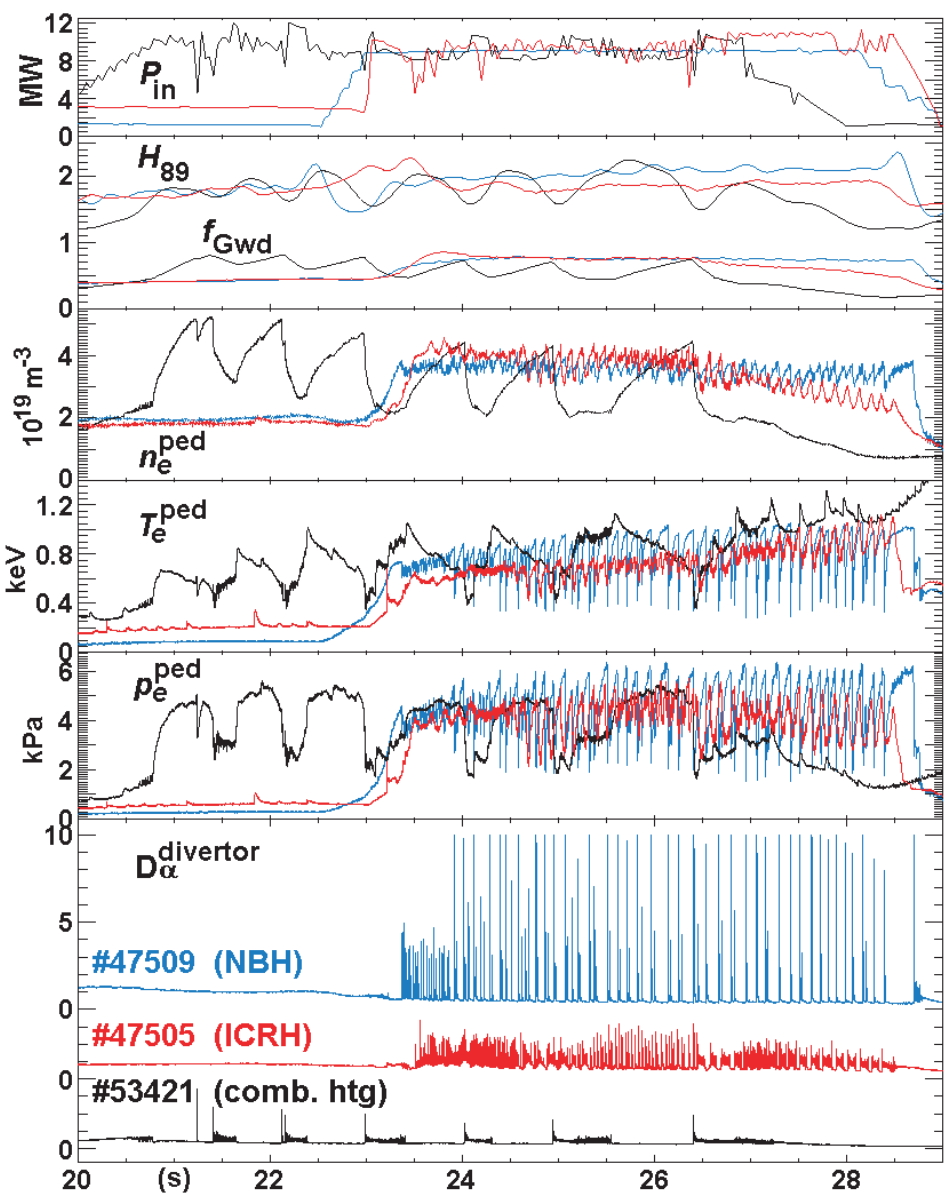

Figure 9. Comparison of three strongly shaped JET MkIIGB plasmas differing only in auxiliary heating scheme: pure NBH (\#47509); pure ICRH (symmetric phasing, \#47505); dominant ICRH (asymmetric phasing) $+\mathrm{NBH}$ (\#53421). Input powers and performance are very similar, but respective ELMs in terms of divertor $\mathrm{D} \alpha$ light are entirely different. Maximum electron pedestal temperatures (ECE) are similar in both cases including beams, but peak pedestal pressure (with FIRI) is lower in the pair including RF. Heating scheme itself therefore seems to influence pedestal and ELM properties.

fluctuations (\#47509). Total energy amplitudes are thus likely to have been reduced with ICRH from the similarly typical size $(\Delta W / W) \approx 8-10 \%$ (\#47509) estimated for the latter from diamagnetic loop measurements. Note the former pulse (\#47505) had only slightly lower power efflux into the edge, $P_{\mathrm{SOL}} \approx 70-80 \% P_{\text {in }}$, compared with $P_{\mathrm{SOL}} \approx 85 \% P_{\text {in }}$ for NBH (\#47509), and actually both plasmas had similar margin over the L- to H-mode power threshold, whether measured according to total heating [30], viz $f_{\text {thresh }} \equiv P_{\text {in }} / P_{\mathrm{L}-\mathrm{H}} \approx 1.2-1.25$, or a modified scaling accounting for radiation from the core [31], viz $f_{\text {thresh }}^{\prime} \equiv P_{\mathrm{SOL}} / P_{\mathrm{L}-\mathrm{H}}^{\prime} \approx 1.25$ (ICRH), $1.4(\mathrm{NBH})$. In the combined heating case (\#53421), however, peak $T_{\mathrm{e}}^{\text {ped }}$ even slightly above the pure NBH level was attained at low density early in each repetitive ELM-free phase, and then declined steadily as density and edge radiation rose. Strikingly, the relative changes corresponded to maintenance of an electron pedestal pressure $p_{\mathrm{e}}^{\text {ped }}$ very close to the pure ICRH peak level (\#47505) once this had been reached, yet without suffering any ELM (\#53421). Only limited charge-exchange data were available in these pulses, but indicated edge ion temperature 
also remained high $T_{\mathrm{i}}^{\mathrm{ped}}>T_{\mathrm{e}}^{\mathrm{ped}}$ with combined heating, and in particular above the level with pure NBH, for which $T_{\mathrm{i}}^{\text {ped }} \approx T_{\mathrm{e}}^{\text {ped }}$. Hence the ion pedestal seemed itself to be preserved, while electron pedestal pressure was nearly constant, as total energy decayed and confinement saturated or rolled over later in each ELM-free period (cf figure 5). Eventually $p_{\mathrm{e}}^{\text {ped }}$ dropped sharply at the following small ELM, then recovered in two stages as $T_{\mathrm{e}}^{\text {ped }}$ and $H_{89}$ began to increase again during the ELM burst, but $n_{\mathrm{e}}^{\text {ped }}$ rose only after its cessation, and the cycle restarted. Both $P_{\mathrm{SOL}}$ and $f_{\text {thresh }}$ fluctuated too throughout this sequence, in fact staying lower around $P_{\mathrm{SOL}} \approx 55-65 \% P_{\text {in }}$ and $f_{\text {thresh }} \approx 1$ in the higher density ELM-free phases, but then rising around the onset of ELMs, before transiently peaking at $P_{\mathrm{SOL}} \approx 90-100 \% P_{\text {in }}$ with $f_{\text {thresh }} \approx 1.5$ in the lower density ELMy interludes. Hence highest, ELM-free performance tended to occur for heating close to the transition threshold, whereas very small ELMs, though at somewhat lower performance, persisted for conditions deeper into H-mode than in either of the pure RF or NB states. A variation with plasma profile shapes is therefore suggested, as core to pedestal peaking increased markedly during each ELMy interval, before flattening to more typical H-mode levels in the ELM-free phases.

Final $n_{\mathrm{e}}^{\text {ped }}$ just prior to an ELMy stage of combined heating (\#53421) was again close to both other cases, but crucially no discernible fall in low $T_{\mathrm{e}}^{\text {ped }}$ occurred at the first ELM in each brief group. Energy expelled was consequently associated only with the efflux of particles, so that it represented a so-called 'minimum' ELM due solely to convective losses [15]. Its estimated size $(\Delta W / W) \approx 3 \%$ (cf section 3 ) is consistent with equivalent events at higher $T_{\mathrm{e}}^{\text {ped }}$ in related NBH plasmas $[13,15]$, reinforcing the potential of the present result (\#53421) as a pointer towards high performance with benign ELMs. Comparisons in figure 9 of alternatively heated plasmas at the same input power and density hence indicate pedestal and ELM properties can be affected by the heating scheme itself.

\section{Summary and discussion}

The EDA H-mode pioneered on Alcator C-Mod [5-7] potentially combines all of the features required for a next-step burning plasma regime, viz high-confinement, high-density and quasistationarity employing RF auxiliary heating, while remaining free from excessive loads onto boundary surfaces due to ELMs. Specifically, persistent high-order coherent fluctuations (QCMs) in the edge seem to avert ELMs by continuously rather than suddenly relaxing pedestal gradients. A globally similar LPCH-mode [8] had also been obtained previously in the Mk0 design of JET. Initial efforts to recover EDA/LPCH states in the JET MkIIGB divertor configuration have matched their essential strong shaping $(\kappa \approx 1.7, \bar{\delta} \approx 0.44)$ and edge safety factor $\left(q_{95} \geqslant 3.7\right)$, and demonstrated excellent sustained coupling of minority fundamental ICRH (up to $P_{\mathrm{ICRH}} \approx 11 \mathrm{MW}$ ). By contrasting such plasmas with comparable confinement and density but formed with the divertor cryopump either on or off, increased peripheral neutral particle sources have been shown to lead to smaller, higher frequency $(\sim 200 \mathrm{~Hz})$ ELMs but without loss of density $\left(f_{\mathrm{Gwd}} \approx 0.8\right)$ or good confinement $\left(H_{89} \approx 1.8\right)$. In other words, this already represents a state of moderated ELMs. However, highest torus gas pressure achieved in JET remains substantially lower than is typical during EDA in C-Mod, and an equivalent recycling regime has not yet been attainable given the usually unsaturated walls of JET discharges. For standard cryopumped operation, adoption of asymmetric $(-\pi / 2)$ phasing of the ICRH antennae has helped to regularize sawteeth and so to improve stability in particular against NTMs [19]. Using combined heating with dominant ICRH, repetitive ELMfree H-mode intervals have then been produced, separated by flurries of only very small ELMs. Each quiescent period exhibits a steady rise in density to $f_{\mathrm{Gwd}} \approx 0.7-0.8$ and edge radiated 
power fraction to $f_{\text {rad }} \approx 1$, while confinement increases to $H_{89}>2$ before saturating or rolling over in the core. Though all properties drop at the subsequent onset of small, compound ELMs, even the largest first one of these seems to be a minimal [15], purely convective fluctuation with total energy size $(\Delta W / W) \approx 3 \%$. Conditions could therefore be approaching a useful regime of tolerable ELMs. In addition, both magnetic pick-ups and multi-channel reflectometry detect coherent fluctuations at two narrow frequencies between $25-60 \mathrm{kHz}$ throughout successive ELM-free periods, the latter density disturbances being localized within the outer 10-20\% of the plasma. Further studies are still required to determine whether these modes occur more generally throughout RFH or other ELM-free H-modes, or could create a steady state, but their toroidal order $n \approx+5-10$ and/or rotation in the ion diamagnetic drift direction are in contrast to QCMs in C-Mod [7], to 'washboard' modes common between ELMs in JET [23], and to features in quiescent H-modes on DIII-D [25, 26]. Differing results for given density $\left(f_{\text {Gwd }} \approx 0.75\right)$ and total input power $(\sim 10 \mathrm{MW})$ from various methods have also shown that additional heating schemes themselves can affect electron pedestal and ELM characteristics. Notably, pedestal temperature and pressure peaks appear to be lower for ICRH than for NBH, provoking smaller, higher frequency ELMs at $\approx 10 \%$ lower confinement $\left(30-50 \mathrm{~Hz}, \mathrm{H}_{89} \approx 1.8\right.$ and $10-15 \mathrm{~Hz}, H_{89} \approx 2.0$, respectively). This seems an extra benefit of the likely heating system in a next-step device.

Presently the reasons for non-appearance of EDA-mode so far in JET remain under study, but both plasma collisionality in the pedestal region and neutral particle density around the edge have been much lower than typify transitions in C-Mod. Collisionless conditions also during LPCH-mode in JET Mk0 suggest that, despite a global resemblance, it may not in fact be the same type of regime. Moreover, plasma density here may have been too low to achieve so-called 'mixed Type I + II' ELMs seen in related JET cases at high triangularity with NB heating [13]. In particular, pedestal density around $\approx 60 \%$ of the Greenwald limit seems to have stayed below the threshold of $\approx 70-75 \%$ reported for such features [13]. Further experiments will aim to address these differences by increasing the initial density prior to entering $\mathrm{H}$-mode, following the approach established on C-Mod, and above all by pursuing identity states reproducing as many of the dimensionless edge and/or core variables of the latter as possible. Comparisons in this form have successfully been made on the DIII-D tokamak [25], while the feasibility of scaling conditions to JET has been shown by earlier trials [32] at very low field, current and power, though not yet with satisfactory matches in terms of $q_{95}$ and shape parameters. As already noted, torus gas density close to that in C-Mod is harder to recover in JET, so by separately matching collisionality, plasma identity tests should simultaneously help to isolate and clarify the influence of edge sources, together with effects of differing divertor geometries. More pairs of ICRH and NBH plasmas at higher density should also check whether their pedestal and ELM properties remain distinct, and perhaps whether 'mixed' ELMs actually depend on specific details of NB heating like deposition profile, fuelling or momentum injection. Another experimental technique could be actively to encourage coherent edge modes by applying external magnetic perturbations with appropriate resonances, or even injecting RF beatwaves favouring the same kind of excitation. This might either seek fluctuations closer to QCMs in C-Mod, or examine the apparently new, oppositely-rotating inter-ELM modes described for combined heating with $-\pi / 2$ antenna phasing. A successful scaling of EDA conditions to JET would ultimately point towards a high performance, benign ELMs regime meeting the integrated requirements of a next-step tokamak. 


\section{Acknowledgments}

This work has been conducted under the European Fusion Development Agreement and was partly funded by Euratom, the UK Department of Trade and Industry, and the US Department of Energy. The authors acknowledge the contribution of the late S J Fielding who suggested and encouraged these experiments on JET.

\section{References}

[1] Matthews G F et al 1999 Nucl. Fusion 3919

[2] McKee G R et al 2000 Phys. Plasmas 71870

[3] Maddison G P et al 2000 Presented at 18th IAEA Fus. Energy Conf. (Sorrento, October 2000) submitted to Nucl. Fusion

[4] Ongena J et al 2001 Plasma Phys. Control. Fusion 43 A11

[5] Takase Y et al 1997 Phys. Plasmas 41647

[6] Snipes J A et al 1997 Proc. 24th EPS Conf. Contr. Fus. and Plas. Phys. (Berchtesgaden, June 1997) vol 21A (II), p 565

[7] Greenwald M et al 1999 Phys. Plasmas 61943

[8] Bureš M et al 1992 Nucl. Fusion 32539

[9] Saibene G et al 1999 Proc. 26th EPS Conf. Contr. Fus. and Plas. Phys. (Maastricht, June 1999) vol 23J, p 97

[10] Horton L D et al 1999 Nucl. Fusion 391

[11] Yushmanov P N et al 1990 Nucl. Fusion 301999

[12] Stober J et al 2001 Nucl. Fusion 411123

[13] Saibene G et al 2001 Proc. 28th EPS Conf. Contr. Fus. and Plas. Phys. (Madeira, June 2001) OR.28

[14] Saibene G et al 1999 Nucl. Fusion 391133

[15] Loarte A et al 2001 Proc. 28th EPS Conf. Contr. Fus. and Plas. Phys. (Madeira, June 2001) P3.005

[16] LaBombard B et al 2001 Phys. Plasmas 82107

[17] Bosch H S et al 1999 J. Nucl. Mater. 266-269 462

[18] Kallenbach A et al 1999 Plasma Phys. Control. Fusion 41 B177

[19] Sauter O et al 2001 Proc. 28th EPS Conf. Contr. Fus. and Plas. Phys. (Madeira, June 2001) OR.08

[20] Snipes J A et al 2001 Proc. 28th EPS Conf. Contr. Fus. and Plas. Phys. (Madeira, June 2001) P1.017

[21] Hastie R J et al 2001 Proc. 28th EPS Conf. Contr. Fus. and Plas. Phys. (Madeira, June 2001) P1.065

[22] Mossessian D et al 2001 Proc. 28th EPS Conf. Contr. Fus. and Plas. Phys. (Madeira, June 2001) P1.056

[23] Smeulders P et al 1999 Plasma Phys. Control. Fusion 411303

[24] Snipes J A et al 2000 42nd APS DPP Meeting (Quebec City, October 2000)

[25] Moyer R A et al 2001 43rd APS DPP Meeting (Long Beach, October 2001)

[26] Doyle E J et al 2001 Plasma Phys. Control. Fusion 43 A95

[27] Connor J W et al 1998 Phys. Plasmas 52687

[28] Sartori R et al 1999 Proc. 26th EPS Conf. Contr. Fus. and Plas. Phys. (Maastricht, June 1999) vol 23J, p 197

[29] LoarteA and Lomas P J Private communications

[30] Ryter F et al 2002 Plasma Phys. Control. Fusion 44 A415

[31] Snipes J A et al 1997 Proc. 24th EPS Conf. Contr. Fus. and Plas. Phys. (Berchtesgaden, June 1997) vol 21A (III), p 961

[32] Christiansen J P et al 1998 Proc. 17th IAEA Fus. Energy Conf. (Yokohama, October 1998) EXP2/02 